\title{
Factors associated with physician follow-up among patients with chest pain discharged from the emergency department
}

\author{
Michael K.Y. Wong, Julie T. Wang MSc, Andrew Czarnecki MD, Maria Koh MSc, Jack V. Tu MD PhD, \\ Michael J. Schull MD MSc, Harindra C. Wijeysundera MD PhD, Ching Lau MD, Dennis T. Ko MD MSc \\ Author audio interview: soundcloud.com/cmajpodcasts/ko-chest-pain
}

Competing interests: None declared.

This article has been peer reviewed.

Correspondence to:

Dennis Ko, dennis.ko@ices .on.ca

CMAJ 2015. DOI:10.1503 /cmaj.141294

\begin{abstract}
- Abstract
Background: Many patients with chest pain do not receive follow-up from a physician after discharge from the emergency department despite significant survival benefit associated with follow-up care. Our objective was to evaluate factors associated with physician follow-up to understand this gap in practice.

Methods: We conducted an observational study involving patients at high risk who were assessed for chest pain and discharged from an emergency department in Ontario between April 2004 and March 2010. We used multivariable logistic regression to determine the association of clinical and nonclinical characteristics with physician follow-up.

Results: We identified 56767 patients, of whom $25.1 \%$ did not receive any follow-up by a physician, $69.0 \%$ were seen by their primary care physician, and $17.3 \%$ were seen by a cardiologist within 30 days. Patients who had medical comor-

bidities and cardiac conditions such as myocardial infarction or heart failure were less likely to have follow-up. In contrast, a previous visit to a primary care physician was associated with the highest odds of having physician follow-up (odds ratio [OR] 6.44, 95\% confidence interval [Cl] 5.91-7.01). Similarly, a previous visit to a cardiologist was strongly associated with follow-up by a cardiologist (OR 3.01, 95\% Cl 2.85-3.17). Patients evaluated in emergency departments with the highest tertile of chest pain volume were more likely to receive follow-up from any physician (OR 1.52, 95\% Cl 1.31-1.77) and from a cardiologist (OR 2.04, 95\% Cl 1.61-2.57).

Interpretation: Nonclinical factors are strongly associated with physician follow-up for patients with chest pain after discharge from the emergency department. However, patients with comorbidities and at higher risk for future adverse events are less likely to receive follow-up care.
\end{abstract}

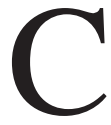
hest pain is one of the most common presenting symptoms in emergency departments. In Canada, about 500000 visits to the emergency department are related to chest pain assessment each year. ${ }^{1}$ Most of these visits result in discharge after excluding a cardiac diagnosis with an immediate risk of adverse effect. ${ }^{2}$ Current clinical guidelines strongly advocate for patients with chest pain who have been discharged from the emergency department to receive outpatient follow-up with a physician within 72 hours for further assessment or treatment, because many patients remain at risk for future events. ${ }^{3}$

Among patients at high baseline cardiovascular risk who were discharged from the emergency department after assessment of chest pain, our group has previously shown significantly reduced hazard of death or myocardial infarction associated with follow-up with either a primary care physician or a cardiologist within 30 days. ${ }^{2}$ At 1-year postassessment, the rate of death or myocardial infarction was $5.5 \%$ among patients who received cardiologist follow-up, $7.7 \%$ with primary care follow-up and $8.6 \%$ with no physician follow-up. ${ }^{2}$ In addition, we found a considerable gap in practice, with 1 in 4 high-risk patients with chest pain failing to follow-up with a physician within 30 days of assessment in Ontario, Canada. ${ }^{2}$ A better understanding of why physician follow-up does not occur in accordance with guidelines is essential to improve the transition of care from the emergency department to home. Thus, the main objective of our study was to evaluate clinical and nonclinical factors associated with physician follow-up among patients with chest pain after discharge from the emergency department. 


\section{Methods}

\section{Data sources}

We obtained data for this study by linking multiple large, administrative, population-based databases in Ontario. The databases have been extensively used in health research and have been validated for the identification of patients with cardiac risk factors. ${ }^{4-8}$ We used the National Ambulatory Care Reporting System database, which contains information regarding emergency department visits, to identify patients and their comorbidities. We used the Ontario Health Insurance Plan physician claims database, which captures information of the services provided by practising physicians in Ontario, to identify physician visits. We used the Canadian Institute of Health Information Discharge Abstract Database to identify comorbidities and procedural interventions. We used the Ontario Registered Persons Database, which contains vital statistics for all Ontarians, to determine rural residency and mortality after the index event. We identified physician specialty through the Institute for Clinical Evaluative Sciences Physician Database.

\section{Study population}

The study cohort included patients at high cardiovascular risk at baseline aged 18 years and older who underwent assessment for chest pain in an Ontario emergency department between Apr. 1, 2004, and Mar. 31, 2010. We identified eligible patients using primary diagnostic codes pertaining to chest pain symptoms as defined by the International Classification of Diseases, 10th revision (codes R07.1-R07.4, I20.0, I20.1, I20.8, I20.88, I20.9). We defined patients as being at high risk at baseline if they had diabetes mellitus, a history of cardiovascular disease or previous cardiac intervention. ${ }^{2}$ We excluded patients who had a subsequent visit to an emergency department for chest pain, who were admitted to hospital for an acute coronary syndrome or who died within 30 days of their assessment, because they might not have had sufficient opportunity for physician follow-up.

\section{Definition of physician follow-up}

Using billing records, we first evaluated followup with any type of physician within 30 days of discharge from an emergency department as our primary outcome. Our secondary outcome was follow-up with a cardiologist within 30 days of discharge from an emergency department.

\section{Clinical and nonclinical predicting factors}

We selected the following clinical factors for evaluation: age, sex, income, rural residency, cardiovascular conditions (diabetes, hyperten- sion, dyslipidemia, chronic atherosclerosis, unstable angina, previous myocardial infarction, previous admission to hospital for heart failure, valvular heart disease, atrial fibrillation, ventricular arrhythmias, other arrhythmias, shock, peripheral vascular disease and cerebrovascular disease) and previous medical conditions (respiratory disease, peptic ulcer disease, rheumatologic disease, hemiplegia or paraplegia, dementia, renal disease, cancer, anemia or blood disease, trauma and depression). We determined income using the postal codes of patients' main residences. ${ }^{9}$ We evaluated care in the emergency department using factors such as cardiology consultation, stress tests, discharge against medical advice and use of health services in the preceding year. In addition, we evaluated the following hospital characteristics: teaching hospital status, availability of cardiac catheterization laboratory and annual emergency department chest pain volume in tertiles (as determined by the annual volume of visits for chest pain at each hospital).

\section{Statistical analysis}

We used descriptive statistics to compare the baseline characteristics of patients who followed up with any physician with those who did not; similarly, we compared those with follow-up by a cardiologist with those who had none. We used the $\chi^{2}$ test to compare categorical variables, 1-way analysis of variance to compare for mean values and the Kruskal-Wallis test to compare median values.

We developed separate multilevel hierarchical logistic regression models, clustered at the hospital level, for any physician follow-up and cardiologist follow-up to assess their independent association with clinical and nonclinical factors. We analyzed all data using SAS version 9.3 (SAS Institute Inc, Cary, NC). All statistical tests were 2-tailed, and we considered $p$ values of less than 0.05 to be significant. This study was approved by the ethics board at Sunnybrook Health Sciences Centre, Toronto, Ontario. Informed consent was exempted in accordance with Ontario law, which permits the use of administrative data for research purposes in prescribed entities.

\section{Results}

\section{Physician follow-up after discharge from the emergency department}

We identified 56767 patients for inclusion in our study. The mean age of the cohort was $66( \pm 15)$ years, and $53.0 \%$ of participants were male. We identified 42535 (74.9\%) patients who received physician follow-up within 30 days of discharge (69.0\% with a primary care physician and $17.3 \%$ 
with a cardiologist), leaving 14232 (25.1\%) patients without follow-up. Figure 1 shows the cumulative percentage of patients who received follow-up over time by type of physician. Within the first week, $46.1 \%$ of patients received follow-up with any type of physician, including $37.3 \%$ with a primary care physician, and $6.1 \%$ with a cardiologist.

\section{Clinical and nonclinical predicting factors}

\section{Factors associated with follow-up by any physician}

Patients aged 50 years and older and those with highsocioeconomic status, diabetes mellitus, hypertension, dyslipidemia, atrial fibrillation or cancer had a significantly higher likelihood of follow-up with any physician (Table 1 and Figure 2). Conversely, rural residents, those with cerebrovascular disease, renal disease or a history of trauma were significantly less likely to receive follow-up. Previous myocardial infarction (odds ratio [OR] $0.84,95 \%$ confidence interval [CI] $0.80-0.88$ ), heart failure (OR $0.89,95 \%$ CI $0.82-$ 0.97 ), hemiplegia or paraplegia (OR $0.61,95 \%$ CI $0.52-0.71)$ and dementia (OR $0.36,95 \%$ CI $0.32-0.41)$ were all strongly associated with a lower likelihood of physician follow-up.

A visit to a primary care physician in the pre-

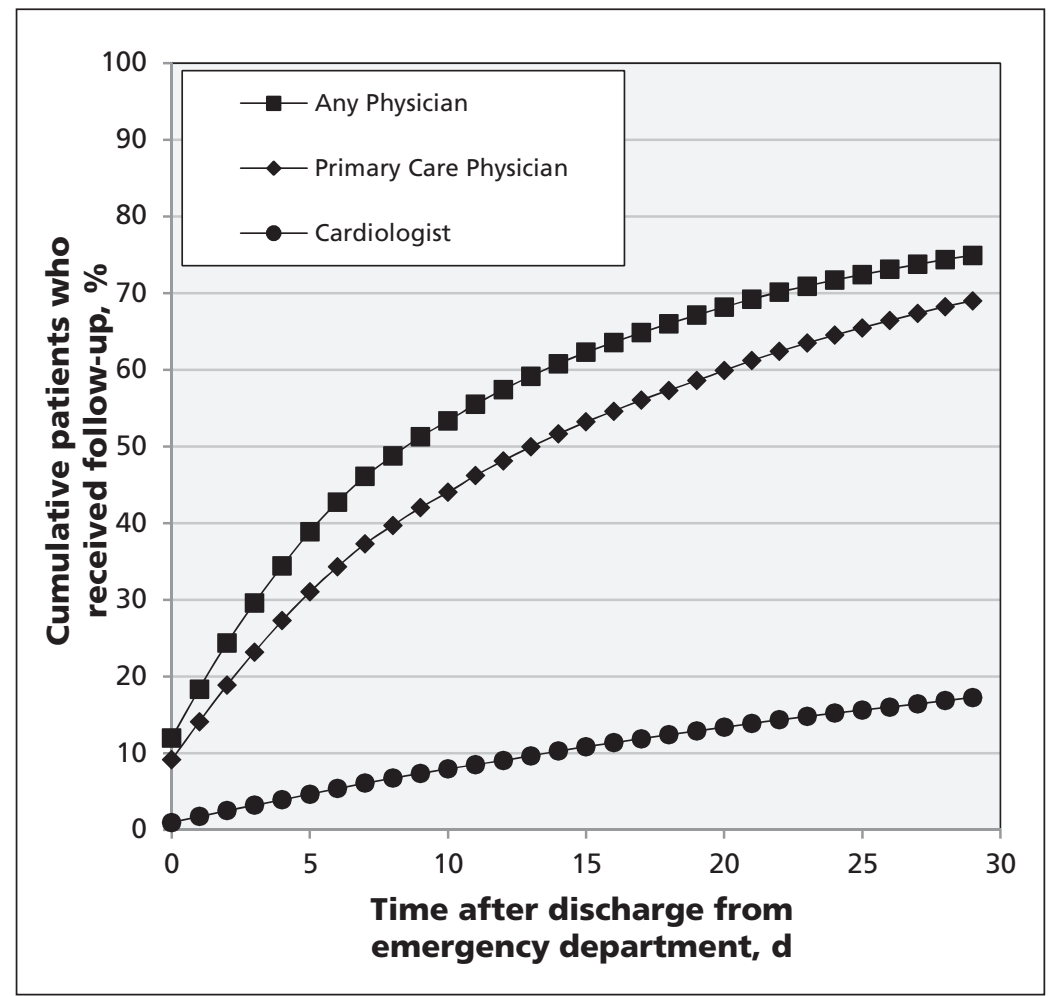

Figure 1: Time course of physician follow-up after discharge from emergency department. The cumulative percentage of patients who received follow-up within 30 days of discharge from the emergency department is shown by physician type. ceding year was associated with the highest likelihood of physician follow-up (OR 6.44, 95\% CI 5.91-7.01). Other factors that were independently associated with follow-up included visiting a cardiologist within the preceding year (OR $1.36,95 \%$ CI 1.29-1.42) and admission to hospital within the preceding year (OR 1.27, 95\% CI 1.22-1.32). Patients discharged from an emergency department with higher chest pain volumes (when compared with the lowest tertile) were more likely to receive follow-up (OR 1.52, 95\% CI 1.31-1.77 for the highest tertile; OR $1.30,95 \%$ CI $1.14-1.50$ for the middle tertile).

\section{Factors associated with follow-up by a cardiologist}

Patients aged 50-69 years, men, and patients with higher socioeconomic status, dyslipidemia, chronic atherosclerosis, previous admission to hospital for heart failure, arrhythmia or ventricular arrhythmias had a significantly higher likelihood of follow-up with a cardiologist (Table 2 and Figure 3). Conversely, patients with medical comorbidities such as diabetes, unstable angina, peripheral vascular disease, cerebrovascular disease, respiratory disease, neurologic disease, cancer, trauma, and depression were significantly less likely to receive follow-up with a cardiologist postdischarge.

Cardiology follow-up was most strongly associated with a cardiologist visit within the preceding year (OR 3.01, 95\% CI 2.85-3.17), admission to hospital during the preceding year (OR 1.30, 95\% CI 1.23-1.36) and discharge from an emergency department with higher chest pain volume (when compared with the lowest tertile: OR 2.04, 95\% CI 1.61-2.57 for the highest tertile; OR 1.46, 95\% CI 1.17-1.81 for the middle tertile).

\section{Patient characteristics stratified by follow-up}

Only $2.5 \%$ of patients received a consultation with a cardiologist in the emergency department, $0.5 \%$ of patients underwent a stress test and $1.5 \%$ of patients left against medical advice (Table 1). The burden of illness, as determined using the Charlson-Deyo score, was significantly higher among patients who did not receive follow-up than among those who did (Table 1).

The clinical characteristics of patients differed substantially between those who received follow-up with a cardiologist and those who did not (Table 2). Patients who received follow-up with a cardiologist had fewer medical comorbidities (Charlson-Deyo score 1.47 in the group with cardiologist follow-up v. 1.68 in the group 
Table 1: Baseline characteristics and factors associated with any physician follow-up within $30 \mathrm{~d}$ of visiting an emergency department with chest pain

\begin{tabular}{|c|c|c|c|c|}
\hline Characteristic & $\begin{array}{c}\text { No physician } \\
\text { follow-up, no. } \\
(\%)^{*} \\
n=14232\end{array}$ & $\begin{array}{c}\text { Any physician } \\
\text { follow-up, no. (\%)* } \\
n=42535\end{array}$ & Unadjusted OR $(95 \% \mathrm{Cl})$ & Adjusted OR+ $(95 \% \mathrm{Cl})$ \\
\hline Age, $y r$, mean $\pm S D$ & $65.90 \pm 16.46$ & $66.68 \pm 14.05$ & & \\
\hline$<50$ (reference) & $2494(17.5)$ & $5335(12.5)$ & 1.00 & 1.00 \\
\hline $50-69$ & $5212(36.6)$ & $17276(40.6)$ & $1.55(1.46-1.64)$ & $1.36(1.28-1.45)$ \\
\hline$\geq 70$ & $6526(45.9)$ & $19924(46.8)$ & $1.43(1.35-1.51)$ & $1.34(1.26-1.43)$ \\
\hline Male sex & $7545(53.0)$ & $22351(52.5)$ & $0.98(0.95-1.02)$ & $0.97(0.93-1.01)$ \\
\hline Rural residency & $3007(21.1)$ & $6601(15.5)$ & $0.69(0.65-0.72)$ & $0.91(0.85-0.98)$ \\
\hline Income quintile 1 (lowest) (reference) & $3733(26.2)$ & $10191(24.0)$ & 1.00 & 1.00 \\
\hline Income quintile 2 & $3070(21.6)$ & $9148(21.5)$ & $1.09(1.03-1.15)$ & $1.04(0.98-1.11)$ \\
\hline Income quintile 3 & $2720(19.1)$ & $8481(19.9)$ & $1.14(1.08-1.21)$ & $1.07(1.01-1.14)$ \\
\hline Income quintile 4 & $2445(17.2)$ & $7742(18.2)$ & $1.16(1.09-1.23)$ & $1.08(1.01-1.15)$ \\
\hline Income quintile 5 (highest) & $2264(15.9)$ & $6973(16.4)$ & $1.13(1.06-1.20)$ & $1.04(0.98-1.11)$ \\
\hline \multicolumn{5}{|c|}{ Cardiac risk factors or previous cardiovascular conditions } \\
\hline Diabetes mellitus & 5066 (35.6) & $15497(36.4)$ & $1.04(1.00-1.08)$ & $1.07(1.02-1.12)$ \\
\hline Hypertension & $9942(69.9)$ & $32680(76.8)$ & $1.43(1.37-1.49)$ & $1.23(1.17-1.29)$ \\
\hline Dyslipidemia & $5044(35.4)$ & $18250(42.9)$ & $1.37(1.32-1.42)$ & $1.14(1.09-1.19)$ \\
\hline Chronic atherosclerosis & $6556(46.1)$ & $20887(49.1)$ & $1.13(1.09-1.17)$ & $1.02(0.97-1.07)$ \\
\hline Unstable angina & $1880(13.2)$ & $5703(13.4)$ & $1.02(0.96-1.08)$ & $0.95(0.89-1.01)$ \\
\hline Previous myocardial infarction & $3979(28.0)$ & $11086(26.1)$ & $0.91(0.87-0.95)$ & $0.84(0.80-0.88)$ \\
\hline Previous admission for heart failure & $980 \quad(6.9)$ & $2786 \quad(6.5)$ & $0.95(0.88-1.02)$ & $0.89(0.82-0.97)$ \\
\hline Valvular heart disease & $651 \quad(4.6)$ & $2305 \quad(5.4)$ & $1.20(1.09-1.31)$ & $1.05(0.96-1.16)$ \\
\hline Arrhythmias & $3553(25.0)$ & $11210(26.4)$ & $1.08(1.03-1.12)$ & $0.92(0.85-0.99)$ \\
\hline Atrial fibrillation & $2291(16.1)$ & $7639(18.0)$ & $1.14(1.08-1.20)$ & $1.18(1.08-1.28)$ \\
\hline Ventricular arrhythmias & $272 \quad(1.9)$ & $879 \quad(2.1)$ & $1.08(0.94-1.24)$ & $1.13(0.96-1.32)$ \\
\hline Shock & $358 \quad(2.5)$ & $1000 \quad(2.4)$ & $0.93(0.83-1.05)$ & $0.99(0.87-1.13)$ \\
\hline Peripheral vascular disease & $893 \quad(6.3)$ & $2854 \quad(6.7)$ & $1.07(0.99-1.16)$ & $1.06(0.98-1.15)$ \\
\hline Cerebrovascular disease & $1830(12.9)$ & $4445(10.5)$ & $0.79(0.75-0.84)$ & $0.91(0.85-0.97)$ \\
\hline \multicolumn{5}{|l|}{ Previous medical conditions } \\
\hline Respiratory disease & $1759(12.4)$ & $5083(12.0)$ & $0.96(0.91-1.02)$ & $0.99(0.93-1.05)$ \\
\hline Peptic ulcer disease & $240 \quad(1.7)$ & $819 \quad(1.9)$ & $1.15(0.99-1.32)$ & $1.09(0.94-1.27)$ \\
\hline Rheumatologic disease & $170 \quad(1.2)$ & $520 \quad(1.2)$ & $1.02(0.86-1.22)$ & $1.10(0.92-1.33)$ \\
\hline Neurologic disease & $1051 \quad(7.4)$ & 1261 & & \\
\hline Hemiplegia or paraplegia & $359 \quad(2.5)$ & $555 \quad(1.3)$ & $0.51(0.45-0.58)$ & $0.61(0.52-0.71)$ \\
\hline Dementia & $724 \quad(5.1)$ & $729 \quad(1.7)$ & $0.33(0.29-0.36)$ & $0.36(0.32-0.41)$ \\
\hline Renal disease & $980 \quad(6.9)$ & $2419 \quad(5.7)$ & $0.82(0.76-0.88)$ & $0.81(0.74-0.89)$ \\
\hline Cancer & $693 \quad(4.9)$ & $2881 \quad(6.8)$ & $1.42(1.30-1.55)$ & $1.35(1.23-1.47)$ \\
\hline Anemia or blood disease & $1230 \quad(8.6)$ & $3414 \quad(8.0)$ & $0.92(0.86-0.99)$ & $0.94(0.87-1.01)$ \\
\hline Trauma & $1335 \quad(9.4)$ & $2848 \quad(6.7)$ & $0.69(0.65-0.74)$ & $0.78(0.72-0.84)$ \\
\hline Depression & $748 \quad(5.3)$ & $2038 \quad(4.8)$ & $0.91(0.83-0.99)$ & $1.04(0.95-1.14)$ \\
\hline Charlson-Deyo comorbidity scoreł & $1.69 \pm 1.65$ & $1.62 \pm 1.62$ & & \\
\hline \multicolumn{5}{|l|}{ Events in the emergency department } \\
\hline Cardiologist consultation & $260 \quad(1.8)$ & $1167 \quad(2.7)$ & $1.52(1.32-1.74)$ & $1.33(1.15-1.54)$ \\
\hline Stress test & $56 \quad(0.4)$ & $(0.5)$ & $1.38(1.03-1.84)$ & $1.29(0.95-1.76)$ \\
\hline Discharged against medical advice & $234 \quad(1.6)$ & $599 \quad(1.4)$ & $0.85(0.73-1.00)$ & $0.92(0.79-1.09)$ \\
\hline \multicolumn{5}{|l|}{ Use of health services in the previous year } \\
\hline Primary care visit & $12194(85.7)$ & $41667(98.0)$ & $8.02(7.39-8.70)$ & $6.44(5.91-7.01)$ \\
\hline Cardiologist visit & $4885(34.3)$ & $19359(45.5)$ & $1.60(1.54-1.66)$ & $1.36(1.29-1.42)$ \\
\hline Admission to hospital & $6706(47.1)$ & $22145(52.1)$ & $1.22(1.17-1.27)$ & $1.27(1.22-1.32)$ \\
\hline \multicolumn{5}{|l|}{ Hospital characteristics } \\
\hline Teaching hospital & $2456(17.3)$ & $7559(17.8)$ & $1.04(0.99-1.09)$ & $0.88(0.74-1.05)$ \\
\hline Cardiac catheterization laboratory & $3912(27.5)$ & $12968(30.5)$ & $1.16(1.11-1.21)$ & $1.13(0.96-1.32)$ \\
\hline \multicolumn{5}{|l|}{ Annual chest pain volume, patients/yr } \\
\hline Low $(<348)$ (reference) & $1189 \quad(8.4)$ & $2242 \quad(5.3)$ & 1.00 & 1.00 \\
\hline Medium (348-1 237) & $3170(22.3)$ & $8188(19.3)$ & $1.37(1.26-1.49)$ & $1.30(1.14-1.50)$ \\
\hline $\operatorname{High}(>1237)$ & $9873(69.4)$ & $32105(75.5)$ & $1.73(1.60-1.86)$ & $1.52(1.31-1.77)$ \\
\hline
\end{tabular}


without, $p<0.001)$. The largest difference in system-level factors was previous visits to a cardiologist; $70.9 \%$ of patients with cardiologist follow-up had previously visited a cardiologist, as opposed to $36.8 \%$ in the group without cardiologist follow-up.

\section{Patient
Age, yr
$50-69$ \\ $\geq 70$}

Male sex

Rural residence

Income quintile

2

3

4

5 (highest)

Comorbidities

Diabetes mellitus

Hypertension

Dyslipidemia

Chronic atherosclerosis

Unstable angina

Previous myocardial infarction

Previous admission to hospital for heart failure Valvular heart disease

Arrhythmias

Atrial fibrillation

Ventricular arrhythmias

Shock

Peripheral vascular disease

Cerebrovascular disease

Previous medical conditions

Respiratory disease

Peptic ulcer disease

Rheumatologic disease

Hemiplegia or paraplegia

Dementia

Renal disease

Cancer

Anemia or blood disease

Trauma

Depression

Events in the emergency department

Cardiologist consultation

Stress test

Discharged against medical advice

Use of health services in the previous year

Primary care visit

Cardiologist visit

Admission to hospital

Hospital characteristics

Teaching hospital

Cardiac catheterization laboratory

Annual chest pain volume in emergency department Medium

High

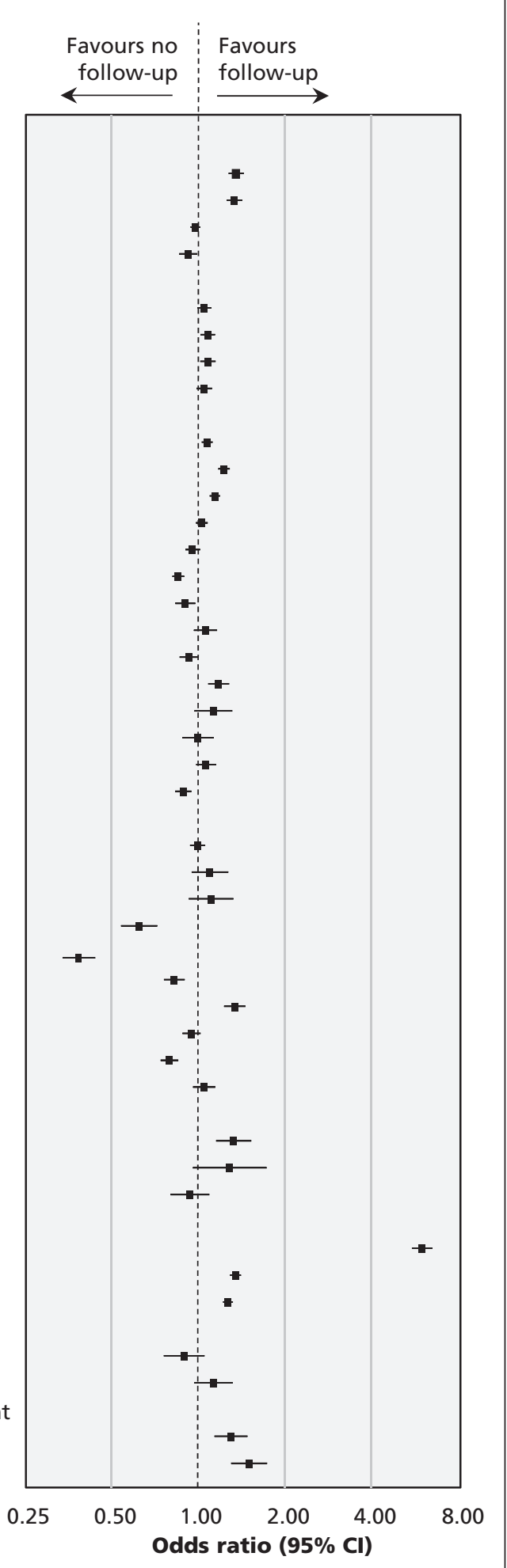

Figure 2: Adjusted odds of physician follow-up within 30 days for adult patients with established cardiovascular disease or diabetes who underwent assessment for chest pain and were discharged from the emergency department during the study period. Baseline demographics, cardiac risk factors and medical conditions, events in the emergency department, use of health services in the previous year and hospital characteristics were included in the model. $\mathrm{Cl}=$ confidence interval. Note: Reference standards presented in Table 1. 
Table 2: Baseline characteristics and factors associated with cardiology follow-up within $30 \mathrm{~d}$ of visiting an emergency department with chest pain

\begin{tabular}{|c|c|c|c|c|}
\hline Characteristic & $\begin{array}{l}\text { No cardiology } \\
\text { follow-up, } \\
\text { no. }(\%)^{*} \\
n=46957\end{array}$ & $\begin{array}{l}\text { Cardiology follow-up, } \\
\text { no. }(\%)^{*} \\
n=9810\end{array}$ & Unadjusted OR $(95 \% \mathrm{Cl})$ & Adjusted OR $(95 \% \mathrm{Cl})$ \\
\hline Age, yr, mean \pm SD & $66.77 \pm 14.95$ & $65.11 \pm 13.32$ & & \\
\hline$<50$ (reference) & 6508 (13.9) & $1321(13.5)$ & 1.00 & 1.00 \\
\hline $50-69$ & $18034(38.4)$ & $4454(45.4)$ & $1.22(1.14-1.30)$ & $1.17(1.08-1.26)$ \\
\hline$\geq 70$ & 22415 (47.7) & $4035(41.1)$ & $0.89(0.83-0.95)$ & $0.96(0.89-1.04)$ \\
\hline Male sex & $24087(51.3)$ & $5809(59.2)$ & $1.38(1.32-1.44)$ & $1.14(1.09-1.20)$ \\
\hline Rural residency & $8590(18.3)$ & $1018(10.4)$ & $0.52(0.48-0.55)$ & $0.98(0.88-1.08)$ \\
\hline Income quintile 1 (lowest) (reference) & $11784(25.1)$ & $2140(21.8)$ & 1.00 & 1.00 \\
\hline Income quintile 2 & $10128(21.6)$ & $2090(21.3)$ & $1.14(1.06-1.21)$ & $1.07(1.00-1.15)$ \\
\hline Income quintile 3 & $9214(19.6)$ & $1987(20.3)$ & $1.19(1.11-1.27)$ & $1.14(1.06-1.22)$ \\
\hline Income quintile 4 & $8369(17.8)$ & $1818(18.5)$ & $1.20(1.12-1.28)$ & $1.10(1.02-1.19)$ \\
\hline Income quintile 5 (highest) & $7462(15.9)$ & $1775(18.1)$ & $1.31(1.22-1.40)$ & $1.19(1.10-1.28)$ \\
\hline \multicolumn{5}{|c|}{ Cardiac risk factors or previous cardiovascular conditions } \\
\hline Diabetes mellitus & 17609 (37.5) & $2954(30.1)$ & $0.72(0.69-0.75)$ & $0.93(0.88-0.98)$ \\
\hline Hypertension & $35329(75.2)$ & $7293(74.3)$ & $0.95(0.91-1.00)$ & $0.99(0.93-1.05)$ \\
\hline Dyslipidemia & 18581 (39.6) & $4713(48.0)$ & $1.41(1.35-1.48)$ & $1.13(1.08-1.19)$ \\
\hline Chronic atherosclerosis & $21700(46.2)$ & $5743(58.5)$ & $1.64(1.57-1.72)$ & $1.12(1.06-1.18)$ \\
\hline Unstable angina & $6145(13.1)$ & $1438(14.7)$ & $1.14(1.07-1.21)$ & $0.92(0.86-0.98)$ \\
\hline Previous myocardial infarction & 12001 (25.6) & $3064(31.2)$ & $1.32(1.26-1.39)$ & $0.96(0.91-1.02)$ \\
\hline Previous admission for heart failure & $3052 \quad(6.5)$ & $714 \quad(7.3)$ & $1.13(1.04-1.23)$ & $1.11(1.01-1.22)$ \\
\hline Valvular heart disease & $2293 \quad(4.9)$ & $663 \quad(6.8)$ & $1.41(1.29-1.54)$ & $1.04(0.94-1.14)$ \\
\hline Arrhythmias & $11787(25.1)$ & $2976(30.3)$ & $1.30(1.24-1.36)$ & $1.11(1.02-1.22)$ \\
\hline Atrial fibrillation & $7980(17.0)$ & $1950(19.9)$ & $1.21(1.15-1.28)$ & $1.04(0.95-1.15)$ \\
\hline Ventricular arrhythmias & $804 \quad(1.7)$ & $347 \quad(3.5)$ & $2.11(1.85-2.39)$ & $1.30(1.11-1.51)$ \\
\hline Shock & $1096 \quad(2.3)$ & $262 \quad(2.7)$ & $1.15(1.00-1.32)$ & $1.03(0.88-1.19)$ \\
\hline Peripheral vascular disease & $3173 \quad(6.8)$ & $574 \quad(5.9)$ & $0.86(0.78-0.94)$ & $0.87(0.79-0.96)$ \\
\hline Cerebrovascular disease & $5576(11.9)$ & $699 \quad(7.1)$ & $0.57(0.53-0.62)$ & $0.77(0.70-0.84)$ \\
\hline \multicolumn{5}{|l|}{ Previous medical conditions } \\
\hline Respiratory disease & $6039(12.9)$ & $803 \quad(8.2)$ & $0.60(0.56-0.65)$ & $0.72(0.66-0.78)$ \\
\hline Peptic ulcer disease & $901 \quad(1.9)$ & $158 \quad(1.6)$ & $0.84(0.71-0.99)$ & $0.91(0.76-1.09)$ \\
\hline Rheumatologic disease & $596 \quad(1.3)$ & $94 \quad(1.0)$ & $0.75(0.61-0.94)$ & $0.90(0.72-1.14)$ \\
\hline Neurologic disease & $2146 \quad(4.6)$ & $166 \quad(1.7)$ & & \\
\hline Hemiplegia or paraplegia & $835 \quad(1.8)$ & $79 \quad(0.8)$ & $0.45(0.36-0.57)$ & $0.71(0.55-0.91)$ \\
\hline Dementia & 1363 & $90 \quad(0.9)$ & $0.31(0.25-0.38)$ & $0.47(0.38-0.59)$ \\
\hline Renal disease & $2842 \quad(6.1)$ & $557 \quad(5.7)$ & $0.93(0.85-1.03)$ & $0.95(0.86-1.06)$ \\
\hline Cancer & $3054 \quad(6.5)$ & $520 \quad(5.3)$ & $0.81(0.73-0.89)$ & $0.89(0.80-0.98)$ \\
\hline Anemia or blood disease & $3988 \quad(8.5)$ & $656 \quad(6.7)$ & $0.77(0.71-0.84)$ & $0.92(0.84-1.01)$ \\
\hline Trauma & $3751 \quad(8.0)$ & $432 \quad(4.4)$ & $0.53(0.48-0.59)$ & $0.72(0.65-0.81)$ \\
\hline Depression & $2491 \quad(5.3)$ & $295 \quad(3.0)$ & $0.55(0.49-0.63)$ & $0.71(0.62-0.80)$ \\
\hline Charlson-Deyo comorbidity score $\ddagger$ & $1.68 \pm 1.64$ & $1.47 \pm 1.56$ & & \\
\hline \multicolumn{5}{|l|}{ Events in the emergency department } \\
\hline Cardiologist consultation & $868 \quad(1.8)$ & $559 \quad(5.7)$ & $1.41(1.29-1.54)$ & $2.03(1.79-2.29)$ \\
\hline Stress test & $203 \quad(0.4)$ & $83 \quad(0.8)$ & $0.84(0.71-0.99)$ & $1.72(1.29-2.29)$ \\
\hline Discharged against medical advice & $716 \quad(1.5)$ & $117 \quad(1.2)$ & $1.41(1.29-1.54)$ & $0.70(0.57-0.87)$ \\
\hline \multicolumn{5}{|l|}{ Use of health services in the previous year } \\
\hline Primary care visit & $44507(94.8)$ & $9354(95.4)$ & $1.13(1.02-1.25)$ & $0.88(0.79-0.98)$ \\
\hline Cardiologist visit & $17285(36.8)$ & $6959(70.9)$ & $4.19(4.00-4.39)$ & $3.01(2.85-3.17)$ \\
\hline Admission to hospital & $23263(49.5)$ & $5588(57.0)$ & $1.35(1.29-1.41)$ & $1.30(1.23-1.36)$ \\
\hline \multicolumn{5}{|l|}{ Hospital characteristics } \\
\hline Teaching hospital & $7750(16.5)$ & $2265(23.1)$ & $0.84(0.71-0.99)$ & $1.12(0.88-1.44)$ \\
\hline Cardiac catheterization laboratory & $13083(27.9)$ & $3797(38.7)$ & $1.41(1.29-1.54)$ & $1.36(1.09-1.70)$ \\
\hline \multicolumn{5}{|l|}{ Annual chest pain volume, patients/yr } \\
\hline Low $(<348)$ (reference) & 3171 & $260 \quad(2.7)$ & 1.00 & 1.00 \\
\hline Medium (348-1237) & $10100(21.5)$ & $1258(12.8)$ & $1.52(1.32-1.75)$ & $1.46(1.17-1.81)$ \\
\hline High $(>1237)$ & $33686(71.7)$ & $8292(84.5)$ & $3.00(2.64-3.41)$ & $2.04(1.61-2.57)$ \\
\hline \multicolumn{5}{|c|}{$\begin{array}{l}\text { Note: } \mathrm{Cl}=\text { confidence interval, } \mathrm{ED}=\text { emergency department, } \mathrm{OR}=\text { odds ratio, } \mathrm{SD}=\text { standard deviation. } \\
\text { *Unless otherwise specified. } \\
\text { † Baseline demographics, cardiac risk factors and medical conditions, events in the emergency department, use of health services in the previous year and hospital } \\
\text { characteristics were included in the model. } \\
\text { ¥Not included in the prediction model because each individual component was adjusted. }\end{array}$} \\
\hline
\end{tabular}




\section{Interpretation}

Ensuring physician follow-up is often difficult for patients whose care is managed in the emergency department, where time is limited and addressing life threatening conditions is the priority. However, integration of services in the emergency

\section{Patient
Age, yr
$50-69$ \\ $\geq 70$ \\ Male sex}

Rural residence

Income quintile

2

3

4

5 (highest)

Comorbidities

Diabetes mellitus

Hypertension

Dyslipidemia

Chronic atherosclerosis

Unstable angina

Previous myocardial infarction

Previous admission to hospital for heart failure

Valvular heart disease

Arrhythmias

Atrial fibrillation

Ventricular arrhythmias

Shock

Peripheral vascular disease

Cerebrovascular disease

Previous medical conditions

Respiratory disease

Peptic ulcer disease

Rheumatologic disease

Hemiplegia or paraplegia

Dementia

Renal disease

Cancer

Anemia or blood disease

Trauma

Depression

Events in the emergency department

Cardiologist consultation

Stress test

Discharged against medical advice

Use of health services in the previous year

Primary care visit

Cardiologist visit

Admission to hospital

Hospital characteristics

Teaching hospital

Cardiac catheterization laboratory

Annual chest pain volume in emergency department Medium

High

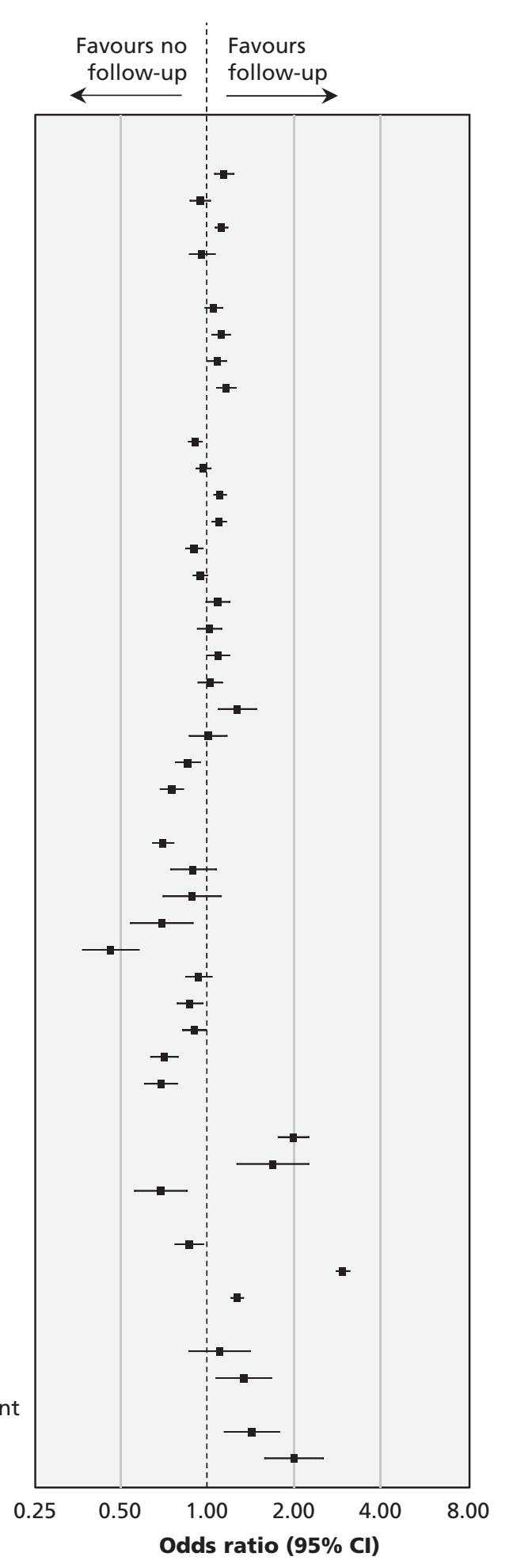

Figure 3: Odds of cardiology follow-up within 30 days for adult patients with established cardiovascular disease or diabetes who underwent assessment for chest pain and were discharged from the emergency department during the study period. Baseline demographics, cardiac risk factors and medical conditions, events in the emergency department, use of health services in the previous year and hospital characteristics were included in the model. $\mathrm{Cl}=$ confidence interval. Note: Reference standards are presented in Table 2. 
department to provide optimal continuity of care is increasingly recognized as a measure of quality of care..$^{10}$ In our study examining predictors of physician follow-up for high-risk patients with chest pain within 30 days of discharge from the emergency department, we identified established access to physician care as perhaps the most important predicting factor. Furthermore, although one would hypothesize that patients with the highest risk at baseline should receive more follow up, we saw a paradoxical pattern that generally favoured healthier patients with fewer comorbidities. In addition, hospital factors played an important role in predicting physician followup; we found that patients seen in emergency departments in the highest tertile of chest pain volume had about $50 \%$ increased odds of receiving postdischarge follow-up with a physician and twice the odds of follow-up with a cardiologist within 30 days compared with those seen in emergency departments in the lowest tertile.

We initially thought that emergency department physicians would risk stratify patients such that those with more medical and cardiac comorbidities would receive more follow-up physician care. However, patients with more medical comorbidities in our cohort were less likely to receive physician follow-up postdischarge. In addition, patient factors associated with any physician follow-up differed from those associated with cardiologist follow-up - poor access for patients with comorbidities was more remarkable for cardiology follow-up, likely reflecting how specialty practice focuses on a primary condition. For example, patients with previous myocardial infarction who presented to the emergency department for chest pain were less likely to receive any physician follow-up and showed no increased odds for cardiology follow-up. This pattern of care has been described as the "treatment-risk paradox," in which lower rates of treatment are given to patients at higher risk of adverse events. ${ }^{11}$ Emergency department physicians would likely not discourage follow-up for patients with more comorbidities, but patients with more serious illness may have less access to follow-up than those who are healthier owing to their underlying conditions and the resulting restrictions in activity. Regardless of the specific reasons as to why physician follow-up was not achieved in patients with the highest baseline risk, we must emphasize that the potential impact of care and treatment is likely highest in these patients ${ }^{11,12}$

We found that systematic factors, such as previous access to health care, were the main driver of follow-up care after a visit to the emergency department rather than clinical characteristics. A visit to a primary care physician within the preceding
12 months was associated with 6-fold increased odds of any physician follow-up, and a previous visit to a cardiologist was associated with 3-fold increased odds of cardiology follow-up. Patients included in our cohort had diabetes or established cardiovascular conditions; however, $5.1 \%$ of these patients had not visited a primary care physician in the year before their chest pain assessment. These patients represent a vulnerable population prone to loss to follow-up and adverse outcomes.

Many studies in cardiology have shown a positive volume-outcome relation where higher volume providers are associated with better patient outcomes. ${ }^{13,14}$ However, the relationship between emergency department volume and care is likely more complex in the Canadian system, given the potential for crowding in emergency departments, which has been shown to adversely affect care and outcomes. ${ }^{15,16} \mathrm{We}$ found that higher emergency department volume was associated with significantly higher odds of physician and cardiology follow-up. Although we did not evaluate patient outcomes, a previous study showed that emergency departments with higher volumes of myocardial infarction miss fewer diagnoses. ${ }^{16}$ We hypothesize that higher volume hospitals are more likely to have established relationships with physicians to improve follow-up care.

Given that many studies have shown the importance of continuity of care during the transitional period between discharge from the emergency department and returning to the community setting, we believe a paradigm shift ensuring follow-up care is needed., ${ }^{2,17,18}$ First, it is important to identify and advocate for vulnerable patients for whom follow-up care is less likely — that is, patients without regular contact with physicians and patients with multiple comorbidities. For these patients, it may be reasonable to schedule follow-up appointments before discharge. This strategy has been supported by several observational studies and randomized control trials to improve physician follow-up and may lead to fewer adverse cardiac events. ${ }^{19,20}$ Methods to facilitate this, however, are uncommon in Canadian emergency departments. We support the development of rapid follow-up or chest pain clinics and online appointment booking for community physicians to allow for the scheduling of prompt follow-up. The participation of community family physicians and cardiologists is critical to ensure the success of such systems.

\section{Limitations}

We used administrative codes to identify a cohort of patient with chest pain. Although previous studies have used similar methods, these codes have not been validated. ${ }^{2,21}$ 
We used physician billing records to determine when follow-up occurred, but we did not have information on the intent of these visits. Thus, some visits may have been routine appointments, and some patients may have scheduled an appointment but were not compliant to follow-up.

We excluded patients with a subsequent visit to an emergency department for chest pain, who were admitted to hospital for acute coronary syndrome or who died within 30 days of assessment to create a stable cohort that had similar opportunities for follow-up. Anticipating the effect of the inclusion of these patients on follow-up rates is difficult.

Finally, our study was performed in the Canadian health care system, and its results may not be fully generalizable outside of this environment.

\section{Conclusion}

The need for follow-up must continue to be strongly emphasized among patients at high risk who present with chest pain to minimize the risk of adverse events. Our study suggests that system factors such as access to care and emergency department annual chest pain volume are the strongest predictors of follow-up rather than patient factors. An improved strategy to ensure follow-up of patients, particularly for those who have not previously seen a physician, is needed to improve the transition of care.

\section{References}

1. NACRS emergency department visits and length of stay by provincelterritory, CTAS level, CHRP peer group, main problem and age group, 2012-2013. Ottawa: Canadian Institute for Health Information;2014.

2. Czarnecki A, Chong A, Lee DS, et al. Association between physician follow-up and outcomes of care after chest pain assessment in high-risk patients. Circulation 2013;127:1386-94.

3. Wright RS, Anderson JL, Adams CD, et al. 2011 ACCF/AHA focused update incorporated into the ACC/AHA 2007 Guidelines for the Management of Patients with Unstable Angina/Non-STElevation Myocardial Infarction: a report of the American College of Cardiology Foundation/American Heart Association Task Force on Practice Guidelines developed in collaboration with the American Academy of Family Physicians, Society for Cardiovascular Angiography and Interventions, and the Society of Thoracic Surgeons. J Am Coll Cardiol 2011;57:e215-367.

4. Tu K, Campbell NR, Chen ZL, et al. Accuracy of administrative databases in identifying patients with hypertension. Open Med 2007; 1:e18-26.

5. Hux JE, Ivis F, Flintoft V, et al. Diabetes in Ontario: determination of prevalence and incidence using a validated administrative data algorithm. Diabetes Care 2002;25:512-6.

6. Williams J, Young W. A summary of studies on the quality of health care administrative databases in Canada [appendix 1]. In: Goel V, Williams J, Anderson G, et al., editors. Patterns of health care in Ontario: the ICES practice atlas. 2nd ed. Ottawa: Canadian Medical Association; 1996:339-45.

7. Juurlink D, Preyra C, Croxford R, et al. Canadian Institute for Health Information Discharge Abstract Database: a validation study. Toronto: Institute for Clinical Evaluative Sciences; 2006.

8. Canadian Institute for Health Information, CIHI data quality study of Ontario emergency department visits for fiscal year 2004-2005 - executive summary. Ottawa: Canadian Institute for Health Information;2008.
9. Wilkins R. Use of postal codes and addresses in the analysis of health data. Health Rep 1993;5:157-77.

10. Schuur JD, Hsia RY, Burstin H, et al. Quality measurement in the emergency department: past and future. Health Aff (Millwood) 2013;32:2129-38.

11. Ko DT, Mamdani M, Alter DA. Lipid-lowering therapy with statins in high-risk elderly patients: the treatment-risk paradox. JAMA 2004;291:1864-70.

12. Ayanian JZ, Landrum MB, Guadagnoli E, et al. Specialty of ambulatory care physicians and mortality among elderly patients after myocardial infarction. $N$ Engl J Med 2002;347: 1678-86.

13. Thiemann DR, Coresh J, Oetgen WJ, et al. The association between hospital volume and survival after acute myocardial infarction in elderly patients. N Engl J Med 1999;340:1640-8.

14. Hannan EL, Racz M, Ryan TJ, et al. Coronary angioplasty volume-outcome relationships for hospitals and cardiologists. JAMA 1997;277:892-8.

15. Schull MJ, Vermeulen M, Slaughter G, et al. Emergency department crowding and thrombolysis delays in acute myocardial infarction. Ann Emerg Med 2004;44:577-85.

16. Schull MJ, Vermeulen MJ, Stukel TA. The risk of missed diagnosis of acute myocardial infarction associated with emergency department volume. Ann Emerg Med 2006;48:647-55.

17. Atzema CL, Austin PC, Chong AS, et al. Factors associated with 90-day death after emergency department discharge for atrial fibrillation. Ann Emerg Med 2013; 61:539-48 e1.

18. Lee DS, Stukel TA, Austin PC, et al. Improved outcomes with early collaborative care of ambulatory heart failure patients discharged from the emergency department. Circulation 2010; 122:1806-14.

19. Baren JM, Boudreaux ED, Brenner BE, et al. Randomized controlled trial of emergency department interventions to improve primary care follow-up for patients with acute asthma. Chest 2006;129:257-65.

20. Zorc JJ, Scarfone RJ, Li Y, et al. Scheduled follow-up after a pediatric emergency department visit for asthma: a randomized trial. Pediatrics 2003;111:495-502.

21. Czarnecki A, Wang JT, Tu JV, et al. The role of primary care physician and cardiologist follow-up for low-risk patients with chest pain after emergency department assessment. Am Heart $J$ 2014;168:289-95.

Affiliations: From the Institute of Clinical Evaluative Sciences, (Wong, Wang, Koh, Tu, Schull, Wijeysundera, Ko); the Department of Medicine, Sunnybrook Health Sciences Centre, University of Toronto (Wong, Czarnecki, Tu, Schull, Wijeysundera, Lau, Ko), Toronto, Ont.

Contributors: All of the authors contributed substantially to the manuscript, drafted the article or revised it critically for important intellectual content, approved the final version submitted for publication and agree to act as guarantors of the work.

Funding: This study was funded by research funding from the Department of Medicine at Sunnybrook Health Sciences Centre and the Sunnybrook Research Institute. Jack Tu is supported by a Canada Research Chair in Health Services Research and a Career Investigator Award from the Heart and Stroke Foundation of Ontario. Michael Schull is supported by a CIHR Applied Chair in Health Services and Policy Research. Harindra Wijeysundera is supported by a Distinguished Clinical Scientist Award from the Heart and Stroke Foundation of Canada. Dennis Ko is supported by a Clinician Scientist Award from the Heart and Stroke Foundation of Ontario.

Disclaimer: There are no financial relationships with any organizations that might have an interest in the submitted work in the previous two years; no other relationships or activities that could appear to have influenced the submitted work. None of the listed organizations endorse this study nor do they have any influence on its publication. None of the authors have any conflicts of interest to declare. The opinions, results and conclusions reported in this paper are those of the authors and are independent from the funding sources. No endorsement by ICES or the Ontario MOHLTC is intended or should be inferred. 